\title{
Very early hypothermia induction in patients with severe brain injury (the National Acute Brain Injury Study: Hypothermia II): a randomised trial
}

\begin{abstract}
Prof Guy L Clifton, MD, Alex Valadka, MD, David Zygun, MD, Prof Christopher S Coffey, PhD, Pamala Drever, RN, Sierra Fourwinds, L Scott Janis, PhD, Elizabeth Wilde, PhD, Pauline Taylor, RN, Kathy Harshman, RN, Adam Conley, MD, Ava Puccio, RN, Prof Harvey S Levin, PhD, Stephen R McCauley, PhD, Prof Richard D Bucholz, MD, Prof Kenneth R Smith, MD, John H Schmidt, MD, James N Scott, MD, Prof Howard Yonas, MD, and Prof David O Okonkwo, MD

Vivian L Smith Center for Neurologic Research, Department of Neurosurgery, The University of Texas Medical School at Houston, Houston, TX, USA (Prof G L Clifton MD, A Valadka MD, P Drever RN, A Conley MD); Seton Brain and Spine Institute, Austin, TX, USA (A Valadka); Department of Critical Care Medicine (D Zygun MD, P Taylor RN) and Department of Radiology (J N Scott MD), University of Calgary, Calgary, AB, Canada; Department of Biostatistics, University of lowa, lowa City, IA, USA (Prof C S Coffey PhD); Department of Biostatistics, School of Public Health, University of Alabama, Birmingham, AL, USA (C S Coffey);Silverwind Research, La Veta, CO, USA (S Fourwinds) ; National Institute of Neurological Disorders and Stroke, Rockville, MD, USA (L S Janis PhD); Baylor College of Medicine, Houston, TX, USA (E Wilde PhD, Prof H S Levin PhD, S R McCauley PhD); Department of Neurosurgery, University of Pittsburgh, Pittsburgh, PA, USA (K Harshman RN, A Puccio RN, Prof D O Okonkwo MD); Division of Neurosurgery, St Louis University, St Louis, MO, USA (Prof R D Bucholz MD, Prof K R Smith MD); Neurological Associates, Charleston, WV, USA (J H Schmidt MD); and Department of Neurosurgery, University of New Mexico, Albuquerque, NM, USA (Prof H Yonas MD)
\end{abstract}

\section{Summary}

Background-The inconsistent effect of hypothermia treatment on severe brain injury in previous trials might be because hypothermia was induced too late after injury. We aimed to assess whether very early induction of hypothermia improves outcome in patients with severe brain injury.

Methods-The National Acute Brain Injury Study: Hypothermia II (NABIS: H II) was a randomised, multicentre clinical trial of patients with severe brain injury who were enrolled within

\footnotetext{
Correspondence to: Prof Guy L Clifton, 6431 Fannin Street, Suite 7.130, Houston, TX 77030, USA, guy.1.clifton@uth.tmc.edu. Contributors

GLC was principal investigator, designed the study, directed the trial, and wrote the first and final drafts of the manuscript. AV, DZ, RDB, KRS, JHS, JNS, HY, and DOO were co-principal investigators and directed the trial at their centres. CSC did the statistical analysis. PD was the overall study coordinator and the Houston coordinator. SF implemented data management. LSJ was the principal investigator in centre recruitment, study design, and acted as a liaison with the study sponsor. EW, HSL, and SRM did patient followup and administered outcome tests. PT, KH, AC, and AP were coordinators and research associates in individual centres.

Study personnel

Data and safety monitoring board M D Walker (chair), W Clarke, D Hanley, B Levin, D Newell, S Starkman; National Institute of Neurological Disorders and Stroke personnel R Hicks (program Director), C S Moy (data and safety monitoring board liaison); Complications monitor M Todd; Medical monitor C Robertson; University of Calgary Health Science Center personnel M Betzner, A Kirkpatrick, W Hader, A Kramer.

Conflicts of interest

We declare that we have no conflicts of interest.
} 
$2.5 \mathrm{~h}$ of injury at six sites in the USA and Canada. Patients with non-penetrating brain injury who were 16-45 years old and were not responsive to instructions were randomly assigned (1:1) by a random number generator to hypothermia or normothermia. Patients randomly assigned to hypothermia were cooled to $35^{\circ} \mathrm{C}$ until their trauma assessment was completed. Patients who had none of a second set of exclusion criteria were either cooled to $33^{\circ} \mathrm{C}$ for $48 \mathrm{~h}$ and then gradually rewarmed or treated at normothermia, depending upon their initial treatment assignment. Investigators who assessed the outcome measures were masked to treatment allocation. The primary outcome was the Glasgow outcome scale score at 6 months. Analysis was by modified intention to treat. This trial is registered with ClinicalTrials.gov, NCT00178711.

Findings-Enrolment occurred from December, 2005, to June, 2009, when the trial was terminated for futility. Follow-up was from June, 2006, to December, 2009. 232 patients were initially randomised a mean of $1.6 \mathrm{~h}$ (SD 0.5) after injury: 119 to hypothermia and 113 to normothermia. 97 patients (52 in the hypothermia group and 45 in the normothermia group) did not meet any of the second set of exclusion criteria. The mean time to $35^{\circ} \mathrm{C}$ for the 52 patients in the hypothermia group was $2.6 \mathrm{~h}$ (SD 1.2) and to $33^{\circ} \mathrm{C}$ was $4.4 \mathrm{~h}(1.5)$. Outcome was poor (severe disability, vegetative state, or death) in 31 of 52 patients in the hypothermia group and 25 of 56 in the normothermia group (relative risk [RR] 1.08, 95\% CI 0.76-1.53; $\mathrm{p}=0.67$ ). 12 patients in the hypothermia group died compared with eight in the normothermia group (RR 1.30, 95\% CI 0.58$2.52 ; \mathrm{p}=0.52)$.

Interpretation-This trial did not confirm the utility of hypothermia as a primary neuroprotective strategy in patients with severe traumatic brain injury.

Funding-National Institute of Neurological Disorders and Stroke.

\section{Introduction}

The results of 23 clinical trials of hypothermia treatment involving 1614 patients with severe brain injury have been inconsistent, ${ }^{1}$ probably because of differences in trial design. Randomised clinical trials of hypothermia for severe brain injury can be divided into those trials in which hypothermia was used to treat raised intracranial pressure ${ }^{2-6}$ and those in which hypothermia was intended as a neuroprotectant, to stop the biochemical cascade after injury. In the former group, hypothermia was started in the first $24 \mathrm{~h}$ after injury but continued through the peak period of raised intracranial pressure (3-5 days) or until intracranial hypertension was resolved..$^{2-6}$ Four of these five studies reported a decrease in mortality rate or in the percentage of patients having a poor recovery ${ }^{2-5}$ accompanied in three by significantly decreased raised intracranial pressure with hypothermia treatment. ${ }^{2-4}$ In the latter group of trials, hypothermia was assessed as a neuroprotectant, similar to its use in cardiac arrest, and reached $33-34^{\circ} \mathrm{C}$ in the first $10 \mathrm{~h}$ after injury and continued for a predefined $24-48 \mathrm{~h}$, irrespective of intracranial pressure. ${ }^{7-9}$ Studies with this design have all failed to show improved outcome with hypothermia treatment.

We previously reported a multicentre $\operatorname{trial}^{7}$ of hypothermia for neuroprotection, in which 392 patients with acute brain injury were randomised to normo thermia or surface-induced hypothermia. Patients in the hypothermia group reached the target temperature of $33^{\circ} \mathrm{C}$ a mean of $8.4 \mathrm{~h}$ (SD 3.0) after injury. Hypothermia did not improve outcome (relative risk [RR] $1 \cdot 0,95 \% \mathrm{CI} 0 \cdot 8-1 \cdot 2)$ and the hypothermia group had a higher rate of hypotension than the normothermia group $(\mathrm{p}=0 \cdot 01){ }^{7}$ However, there was some weak evidence of improved outcomes in patients who were hypothermic on admission and treated with continued hypothermia compared with those in the normothermia group $(\mathrm{p}=0.09) .{ }^{10} \mathrm{We}$ therefore concluded that hypothermia might have been started too late and that hypotension might have affected the results. In the present study, we aimed to assess whether very early 
induction of hypothermia in patients with severe traumatic brain injury improved outcome at 6 months.

\section{Methods}

Patients

The National Acute Brain Injury Study: Hypothermia II (NABIS: H II) was a multicentre randomised trial of patients with severe brain injury who received either early cooling to $33^{\circ} \mathrm{C}$ maintained for $48 \mathrm{~h}$ or treatment at normothermia. Patients were enrolled either during transport to the hospital or in the emergency department. The enrolment window was increased from $2 \mathrm{~h}$ to $2.5 \mathrm{~h}$ after injury in December, 2006, to increase enrolment.

Inclusion criteria were age 16-45 years, non-penetrating brain injury, and not responsive to instructions. There were two sets of exclusion criteria. The initial set of inclusion and exclusion criteria were measured in the field or upon arrival at the emergency department but before resuscitation and trauma assessment in the emergency department. At randomisation, patients were excluded if they had suspected pregnancy, systolic blood pressure less than $110 \mathrm{~mm} \mathrm{Hg}$, diastolic blood pressure less than $60 \mathrm{~mm} \mathrm{Hg}$, sustained heart rate greater than 120 beats per minute, or if they could not be reached by study-affiliated personnel within $2.5 \mathrm{~h}$ of injury. The second set of exclusion criteria were measured after complete assessment and resuscitation. At this stage, patients were further assessed for the presence of a severe brain injury (Glasgow coma scale score of 3-8 after resuscitation) without life-threatening associated injuries. Exclusion criteria were Glasgow coma scale score of 3 with nonreactive pupils, Glasgow coma scale score of 7-8 with normal brain CT scan, inability to measure an accurate Glasgow coma scale score, abbreviated injury severity score of 4 or greater for organs other than the brain, ${ }^{11}$ systolic blood pressure less than 110 $\mathrm{mm} \mathrm{Hg}$, diastolic blood pressure less than $60 \mathrm{~mm} \mathrm{Hg}$, persistent hypoxia (oxygen saturation $<94 \%$ ), or a positive pregnancy test.

Consent was waived unless a family member was immediately able to provide consent. The trial protocol and the decision to waive consent were approved by institutional review and ethics boards of each participating centre.

\section{Randomisation and masking}

Patients were randomly assigned (1:1), stratified by centre, to hypothermia or normothermia. The randomisation sequence was generated by the study biostatistician with a random number generator. The secretary at the trial coordinating centre (University of Texas Medical School at Houston, Houston, TX, USA) placed treatment assignments into numbered opaque envelopes, which were sealed and mailed to each trial centre. Patients were enrolled at six centres: University of Texas Health Science Center at Houston, TX, USA; University of Calgary, AB, Canada; University of Pittsburgh, Pittsburgh, PA, USA; St Louis University, St Louis, MO, USA; Charleston Area Medical Center, Charlestown, WV, USA; and University of New Mexico, Albuquerque, NM, USA. Randomisation was done at each trial centre by study nurses after opening sequentially numbered envelopes. For eligible patients who were enrolled during transport to the hospital, the emergency service personnel contacted the study nurse at the local trial centre by telephone, who informed the emergency service personnel of the treatment assignment.

Investigators who assessed the outcome measures were masked to treatment allocation. Emergency service personnel, study nurses involved in randomisation, and personnel who managed the patients were unmasked to treatment allocation. 


\section{Procedures}

Temperatures below $35^{\circ} \mathrm{C}$ are associated with increased mortality in patients with multiple trauma. ${ }^{12}$ Therefore, patients who were randomised to hypothermia were maintained at $35^{\circ} \mathrm{C}$ by intravenous instillation of up to $2 \mathrm{~L}$ of cold crystalloid and application of wet sheets or gel packs until completion of the trauma assessment. Those assigned to normothermia were maintained at $37^{\circ} \mathrm{C}$.

Patients who did not meet any of the second set of exclusion criteria and who were randomly assigned to the hypothermia group were cooled to $33^{\circ} \mathrm{C}$ by the Arctic Sun Temperature Management System (surface cooling; Medivance, Louisville, CO, USA), use of room temperature ventilated air, continuation of chilled intravenous crystalloid, and gastric lavage with cold water. Patients in the hypothermia group were maintained at $33^{\circ} \mathrm{C}$ for $48 \mathrm{~h}$ and then rewarmed by $0.5^{\circ} \mathrm{C}$ every $2 \mathrm{~h}$, regardless of levels of intracranial pressure. Patients randomly assigned to normothermia who did not meet any of the second set of exclusion criteria were maintained at $37^{\circ} \mathrm{C}$. Patients who were initially randomised but who met the second set of exclusion criteria were not given any further trial treatment.

Morphine was administered to all patients for at least $72 \mathrm{~h}$ at a dose of $0 \cdot 05-0 \cdot 10 \mathrm{mg} / \mathrm{kg} / \mathrm{h}, \mathrm{a}$ lower dose than in our previous study, ${ }^{7}$ to reduce the risk of hypotension. Pancuronium bromide was administered to patients assigned to hypothermia for at least $72 \mathrm{~h}$ and to those assigned to normothermia as needed. Intermittent doses of fentanyl $(1.03 \mu \mathrm{g} / \mathrm{kg})$ were administered to all patients for pain management. Phenytoin was administered to all patients at $20 \mathrm{mg} / \mathrm{kg}$ followed by maintenance doses for 7 days. Blood gases were not corrected for body temperature. Low serum potassium and magnesium concentrations were treated with intravenous replacement, except preceding and during rewarming. The protocol stated that partial pressure of arterial carbon dioxide less than $30 \mathrm{~mm} \mathrm{Hg}$ should be avoided where possible. ${ }^{13}$

Bladder temperature was used to guide temperature management. All patients underwent monitoring with ventricular or intraparenchymal catheters, the Licox Brain Oxygen Monitoring System (Integra LifeSciences, Plainsboro, NJ, USA), and pulmonary artery catheters. Brain oxygen tensions were not used to guide management. Temperatures over $38^{\circ} \mathrm{C}$ were treated with paracetamol and cooling blankets, but other than these methods, no devices were used to maintain normothermia.

At randomisation, patients were assessed for prehospital hypotension (a directly measured systolic blood pressure of less than $110 \mathrm{~mm} \mathrm{Hg}$ or a diastolic blood pressure of less than 60 $\mathrm{mm} \mathrm{Hg}$ ) and prehospital hypoxia (presence of agonal respirations, cyanosis, or oxygen saturation less than $94 \%$ by pulse oximetry). The abbreviated injury severity scores for each body system were measured upon admission for all patients and from them an aggregate injury severity score, which quantifies the overall level of anatomic injury, was calculated. ${ }^{14}$ The injury severity score includes all organ systems, including the head. Rectal, oesophageal, or bladder temperatures were measured and recorded upon arrival to the emergency department. Intravenous fluids (excluding blood products and insensible losses) that were administered in the first $96 \mathrm{~h}$ after injury were also recorded.

After admission to hospital, doses of drugs that patients received, the daily therapeutic intervention score, the daily therapeutic intensity level, and caloric intake on day 5 were recorded. The therapeutic intervention scoring system permits quantitative comparison of the number and intensity of interventions in patients receiving intensive care. ${ }^{15} 49$ interventions are scored with a range of 1-4 points per intervention, with higher scores suggesting a greater number and intensity of interventions. The therapeutic intensity level quantifies the effects of treatment on the analysis of levels of intracranial pressure ${ }^{16}$-for 
example, to distinguish between levels of intracranial pressure maintained with sedation alone and the same levels achieved with barbiturate coma. This scale ranges from 0 to 15 , with higher values suggesting more treatment. The Glasgow outcome scale was measured at 3 months and 6 months after injury and the disability rating scale was measured at 2 weeks, 4 weeks, 3 months, and 6 months after injury.

The primary hypothesis was that the induction of hypothermia will reduce the percentage of patients with poor outcomes at 6 months after injury compared with normothermia. The primary outcome was the Glasgow outcome scale score 6 months after injury, adjusted for age at enrolment and baseline Glasgow coma scale score. Good recovery and moderate disability were designated as favourable outcomes; severe disability, a vegetative state, and death as poor outcomes.

We also recorded the occurrence of 58 complications categorised as critical and non-critical for the duration of hospitalisation. Neurological complications included increased intracranial pressure, decreased cerebral perfusion pressure, hydrocephalus, and new haemorrhages. Cerebral perfusion pressure is the difference between mean arterial pressure and intracranial pressure. Increased intracranial pressure was defined as intracranial pressure greater than $20 \mathrm{~mm} \mathrm{Hg}$ for 2 or more consecutive hours. Critically increased intracranial pressure was defined as intracranial pressure greater than $30 \mathrm{~mm} \mathrm{Hg}$ for $30 \mathrm{~min}$ or greater than $40 \mathrm{~mm} \mathrm{Hg}$ for $5 \mathrm{~min}$, unresponsive to maximum medical management, and that requires barbiturates. Decreased cerebral perfusion pressure was defined as cerebral perfusion pressure less than $60 \mathrm{~mm} \mathrm{Hg}$ for 2 or more consecutive hours and critically decreased cerebral perfusion pressure as less than $50 \mathrm{~mm} \mathrm{Hg}$ at any time. Hydrocephalus was defined as an enlargement of ventricles on CT scan clinically interpreted to be hydrocephalus. New subdural, epidural, intraventricular, and intraparenchymal haemorrhages were those occurring after admission.

Infectious complications were pneumonia, urinary tract infections, bloodstream infections, sinusitis, surgical site infections, ventriculitis, meningitis, asymptomatic bacteriuria, positive culture of catheter tip, fever of unknown origin, and soft tissue infections. Miscellaneous complications were deep vein thrombosis, pneumothorax, decubitus ulcer, acute respiratory distress syndrome, pulmonary embolism, acute renal failure, aspiration, hypothermia other than during induced hypothermia, multiple organ dysfunction syndrome, syndrome of inappropriate antidiuretic hormone, hypoxaemia, and pancreatitis. Cardiovascular complications were hypotension and cardiac arrest. Finally, bleeding complications were haemothorax and gastrointestinal bleeding.

\section{Statistical analysis}

On the basis of a previous trial, ${ }^{7}$ the planned sample size of 240 patients who received $48 \mathrm{~h}$ of hypothermia at $33^{\circ} \mathrm{C}$ or were treated at normothermia would detect a $17 \%$ difference in the percentage of patients with poor outcomes with $80 \%$ power. A single interim analysis of outcome and complications was planned at the midpoint of the trial-ie, after accrual of 120 patients. Stopping rules stated that the trial should be terminated at the interim analysis if there was less than a $20 \%$ chance of confirming the primary hypothesis. However, accrual in the trial was 2 years behind schedule because few centres could be found internationally that had a sufficient number of eligible patients and were also both capable of and willing to participate in the trial. Personnel from the National Institutes of Health (NIH) were concerned about the feasibility of extending funding of the trial for another 2 years without an interim analysis and members of the data safety and monitoring board were concerned about slow accrual and patient safety. Their worries about safety were heightened by a randomised clinical trial ${ }^{17}$ in June, 2008, that reported weak evidence of adverse outcomes in children with severe brain injury treated with hypothermia $(\mathrm{p}=0 \cdot 14)$. Accordingly, 
members of the data safety and monitoring board and the NIH decided to do the planned interim analysis in April, 2009.

Comparisons of prognostic factors, medical treatments, laboratory data, and complications between the two groups were done with a $t$ test, $\chi^{2}$ test, or Fisher's exact test as appropriate. Complication rates within groups were compared by a Poisson regression model. A p value of less than or equal to 0.05 was deemed significant.

The primary analysis was measured by a two-sided test. Percentages in each group were compared using a generalised linear model with a binomial distribution and log-link function, with admission age and baseline Glasgow coma scale score as covariates. The primary analysis was a modified intention-to-treat analysis, which included all randomised patients who met the second set of criteria. For patients with no 6-month Glasgow outcome scale score, values were imputed from 3-month values or disability rating scale scores at $2-4$ weeks on the basis of a model we developed with data from our previous clinical trial ${ }^{7}$ (webappendix).

In September, 2008, 9 months before the interim analysis was done, we presented detailed plans of four subgroup analyses to the data safety and monitoring board. The first time that anyone had access to unmasked outcome data was at the time of the interim analysis. The four subgroup analyses assessed hypothermia treatment by subgroup interactions, which were, as with the primary analysis, adjusted for age at enrolment and baseline Glasgow coma scale score. The analyses were done by age at screening ( $\$ 0$ years $v s \geq 31$ years), Glasgow coma scale score (3-4 vs 5-8), centre (Houston $v s$ other sites), and patients with haematomas that were surgically removed within the first $24 \mathrm{~h}$ after injury versus those without (diffuse brain injury).

This trial is registered with ClinicalTrials.gov, NCT00178711.

\section{Role of the funding source}

The sponsor of the study had no role in the data collection, data analysis, data interpretation, or writing of the report; however, the sponsor was involved in the study design and chose the timing of the interim analysis. The corresponding author had full access to all the data in the study and had final responsibility for the decision to submit for publication.

\section{Results}

Enrolment occurred from December, 2005, to June, 2009, with follow-up from June, 2006, to December, 2009. 49 of 97 patients were enrolled at the University of Texas Health Science Center at Houston. All but three patients were randomly assigned with waiver of consent. The interim analysis was done after 97 patients had been randomly assigned to a treatment and the trial was terminated for futility in June, 2009.

232 patients were randomly assigned to hypothermia $(n=119)$ or normothermia $(n=113$; figure 1) a mean of $1.6 \mathrm{~h}(\mathrm{SD} 0.5)$ after injury. All patients assigned to hypothermia received the hypothermia intervention; none assigned to normothermia received hypothermia. Of 232 patients who were randomised, 135 (67 hypothermia and 68 normothermia) were excluded by the second set of enrolment criteria and 97 remained on their allocated treatment (figure 1). The three main reasons for exclusion by the second set of criteria were Glasgow coma scale score greater than 8 (75 patients), life-threatening injuries (43), and Glasgow coma scale score of 3 with non-reactive pupils (17). Some patients had multiple reasons for exclusion. During the time interval from first temperature measurement to exclusion by the second set of criteria (mean $69 \mathrm{~min}$ [SD 35]), in the 119 patients who were assigned to 
hypothermia their temperature decreased by a mean of $0 \cdot 21^{\circ} \mathrm{C}(\mathrm{SD} 0 \cdot 9)$, whereas in the 113 assigned to normothermia their temperature increased by $0 \cdot 17^{\circ} \mathrm{C}(1 \cdot 1 ; \mathrm{p}=0 \cdot 05)$.

Of the 135 patients who were randomised but subsequently excluded,, there were no significant differences in prognostic variables (age, Glasgow coma scale, injury severity score, and first temperature) or in prothrombin time, partial thromboplastin time, volume of blood products administered, or the number of patients receiving blood products (data not shown). The only individual complications that differed in frequency of occurrence between groups were episodes of intracranial pressure greater than $20 \mathrm{~mm} \mathrm{Hg}$ for 2 consecutive hours ( 82 episodes in the patients assigned to hypothermia vs 30 episodes in those assigned to normothermia; $\mathrm{p}=0.002$ ) and episodes of cerebral perfusion pressure less than $60 \mathrm{~mm} \mathrm{Hg}$ for 2 consecutive hours ( 31 vs one; $\mathrm{p}<0 \cdot 0001$ ).

There were no differences in the outcome of patients who were randomised and excluded by the second set of exclusion criteria (67 in the hypothermia group and 68 in the normothermia group). At discharge from acute-care hospitals, the distribution of the Glasgow coma scale score in the hypothermia group was zero for score 3-4, three for 5-8, four for 9-12, and 50 for $13-15$; and in the normothermia group it was zero for score $3-4$, zero for $5-8$, nine for 9-12, and 49 for $13-15(\mathrm{p}=0 \cdot 62)$.

Of the 97 patients who did not meet any of the second set of exclusion criteria, 52 had been randomly assigned to hypothermia and 45 to normothermia (figure 1 ). The characteristics of the patients were similar between groups (table 1), except that patients in the normothermia group were older $(\mathrm{p}=0.03)$; this difference was driven by four patients in the normothermia group and one person in the hypothermia group who were outside the study's age range. Mean first temperature was the same in both groups. 46 patients in the hypothermia group received a mean of $1997 \mathrm{~cm}^{3}$ (SD 659) of chilled crystalloid (six patients were already below $35^{\circ} \mathrm{C}$ when randomly assigned and their temperature was maintained by the Arctic Sun device).

In the time from first temperature measurement to inclusion by the second set of criteria (mean 24 min [SD 26]), the temperature in the 52 patients in the hypothermia group decreased by a mean of $0.33^{\circ} \mathrm{C}$ (SD 0.9), whereas in the 45 patients in the normothermia group it increased by $0 \cdot 31^{\circ} \mathrm{C}(1 \cdot 4 ; \mathrm{p}=0 \cdot 01)$. Twice as much time was required to identify whether criteria for exclusion were present for those who were randomised and ultimately excluded than for those who were randomised and ultimately included. Whereas identifying patients who had no exclusions (particularly life-threatening associated injuries) was relatively straightforward, more protracted work-ups were usually required for those who were ultimately judged to meet one of the second set of exclusion criteria.

Mean time to reach $35^{\circ} \mathrm{C}$ in patients in the hypothermia group was $2.6 \mathrm{~h}$ (SD 1.2) after injury, and the mean time to $33^{\circ} \mathrm{C}$ was $4.4 \mathrm{~h}(1.5)$. Patients in the hypothermia group were rewarmed over a mean of $17 \cdot 2 \mathrm{~h}$ (SD 3.3). Figure 2 shows the mean hourly temperature in both treatment groups for the first $96 \mathrm{~h}$. Mean temperature for the 48 -h period of hypothermia in the hypothermia group was $33 \cdot 1^{\circ} \mathrm{C}$ (SD 0.6) and for the concurrent 48 -h period in the normothermia group it was $37 \cdot 2(0 \cdot 4 ; \mathrm{p}<0 \cdot 0001)$.

6-month outcome data were available from 89 patients. The 6-month Glasgow outcome scale score was imputed from the 3-month Glasgow outcome scale score for six patients and from the disability rating scale at 4 weeks for one patient and at 2 weeks for another. The classification accuracy of the 3-month Glasgow outcome scale was $90.1 \%$ and for the disability rating scale at 4 weeks after injury it was $87 \cdot 2 \%$. 
We did two separate analyses-one including all 97 patients and one with only data from the 89 patients with 6-month Glasgow outcome scale scores. The results of the two analyses were indistinguishable, so we present only the results of the analysis that included all 97 patients. There was no significant difference in the number of patients in the hypothermia group who had poor outcomes compared with the normothermia group ( $\mathrm{p}=0.67$; table 2$)$. We also identified no significant difference in mortality between groups $(\mathrm{p}=0.52)$.

Table 3 shows the medical interventions that patients received after hospitalisation. During the first $96 \mathrm{~h}$, compared with patients in the normothermia group, patients in the hypothermia group received more interventions for raised intracranial pressure, as shown by the difference in daily therapeutic intensity level ( $\mathrm{p}=0.002)$; and had more interventions in total, as shown by the higher daily therapeutic interventions score in the intensive care unit $(\mathrm{p}=0.0006$; table 3$)$. Patients in the hypothermia group seemed to have negative cumulative fluid balances less often ( $\mathrm{p}=0.08)$ and had higher cumulative fluid balances in the first $96 \mathrm{~h}$ $(\mathrm{p}=0.01)$ than patients in the normothermia group.

Table 4 reports laboratory data. Patients in the hypothermia group had slight, but significant, decreases in mean serum potassium $(\mathrm{p}=0.0005)$ and increases in mean partial thromboplastin time ( $\mathrm{p}=0.004)$. There was no difference between groups in brain oxygen tension below 6 $\mathrm{mm} \mathrm{Hg}$, which has been associated with poor outcomes from severe brain injury..$^{18}$ Patients in the hypothermia group were more likely than those in the normothermia group to have an occurrence of partial pressure of arterial carbon dioxide less than $30 \mathrm{~mm} \mathrm{Hg}(\mathrm{p}=0.02)$.

Table 5 reports complications or critical complications. There were no significant differences in the percentage of patients with any individual complication or group of complications, whether critical or non-critical, between groups. The only complication that was increased in frequency in the hypothermia group compared with the normothermia group was episodes of increased intracranial pressure ( $\mathrm{p}=0.003)$, which resulted in a significant increase in the total rate of complications in patients in the hypothermia group $(\mathrm{p}=0 \cdot 01)$.

In the subgroup analyses, there was no interaction between treatment and centre $(\mathrm{p}=0 \cdot 70)$, baseline Glasgow coma scale score $(\mathrm{p}=0.24)$, or age at screening $(\mathrm{p}=0.99)$. However, there was a significant interaction between treatment and presence of surgically removed haematoma compared with diffuse brain injury ( $p=0 \cdot 001)$. Thus, we assessed the effect of hypothermia treatment within each subgroup (table 2). Patients who underwent surgical removal of intra cranial haematomas and had hypothermia had significantly fewer poor outcomes than patients who had normothermia $(\mathrm{p}=0 \cdot 02)$. However, we found some weak evidence that patients with diffuse brain injury who were treated with hypothermia had more poor outcomes than patients in the normothermia group ( $\mathrm{p}=0 \cdot 09$ ). Additionally, there was weak evidence of increased mortality in patients with diffuse brain injury who were treated with hypothermia compared with those who received normothermia $(p=0 \cdot 08)$. The 37 patients with diffuse brain injury who were treated with hypothermia had 149 episodes of critically raised intracranial pressure compared with 23 episodes in the 32 patients in the normothermia group $(\mathrm{p}=0.003)$.

Two patients in the hypothermia group had epidural haematomas and 13 had subdural or intracerebral haematomas. Three patients in the normothermia group had epidural haematomas and ten had other types. Patients with surgically removed haematomas who were treated with hypothermia had 52 episodes of critically raised intracranial pressure compared with 41 episodes in the normothermia group ( $\mathrm{p}=0 \cdot 90)$. Three patients with diffuse injury had craniectomies for intracranial pressure control (two in the hypothermia group and one in the normothermia group). Two patients in the hypothermia group who had surgically 
removed haematomas and none in the normothermia group had craniectomies for intracranial pressure control. Bone flaps were left out at craniotomy in five of 15 patients in the hypothermia group and in six of 13 patients in the normothermia group, but the difference was not significant in a post-hoc analysis $(\mathrm{p}=0 \cdot 49)$.

\section{Discussion}

We found no significant difference in outcome in patients treated with hypothermia compared with those treated with normothermia; however, patients in the hypothermia group did have a significantly higher number of episodes of increased intracranial pressure than those in the normothermia group. Additionally, we identified a difference in outcome between the subgroup of patients with diffuse brain injury and those with surgically evacuated haematomas.

The finding of raised intracranial pressure with hypothermia treatment is unique to this study (panel). ${ }^{2-10}$ On the basis of an increased rate of hypothermia-induced hypotension in our previous study, ${ }^{7}$ we used fluid expansion, low-dose morphine, and vasopressors to reduce the rate of hypotension. In our previous study, ${ }^{7} 53 \%$ of patients in the hypothermia group had a mean arterial pressure of less than $70 \mathrm{~mm} \mathrm{Hg}$ in the first $96 \mathrm{~h}$ compared with $39 \%$ in the present study (data not shown). Rewarming rates (about $17 \mathrm{~h}$ ) were the same in both studies and do not explain the difference in intracranial pressure increases in the two studies. The most likely explanation for the difference in intracranial pressure is that our more rigorous measures to reduce hypotension in this trial resulted in higher levels of critically raised intracranial pressure during hypothermia, although the finding occurred in patients with diffuse injury rather than those with evacuated haematomas.

One potentially contributing factor in the different outcomes between patients with diffuse injury and those with surgically removed haematomas could be the surgical treatment of patients with haematomas, in which bone flaps were left out in one third of patients in the hypothermia group and almost half of those in the normothermia group. The absence of bone flaps could suppress the rebound increased intracranial pressure with hypothermia treatment. There were no differences in the types of haematomas between treatment groups that would explain the improved outcome with hypothermia treatment of patients with evacuated haematomas.

We believe that the most important finding in this study is the difference in outcomes in patients with diffuse brain injury compared with those with surgically evacuated haematomas. The difference in direction of the treatment effect between the two groups was highly significant $(\mathrm{p}=0 \cdot 001)$. One explanation might be the differing pathophysiology of patients with haematomas and those with diffuse brain injury. Patients with surgically removed haematomas have a higher incidence of raised intracranial pressure. ${ }^{19}$ In experimental models, ischaemia occurs during haematoma expansion followed by reperfusion after removal, ${ }^{20}$ which is similar in pathophysiology to that of patients with cardiac arrest-a group that has been successfully treated with hypothermia. ${ }^{21}$

Experimentally, intra-ischaemic hypothermia after haematoma removal is associated with improved outcome. ${ }^{22}$ However, diffuse brain injury is not characterised by ischaemia in invitro studies. ${ }^{23}$

The limitations of this clinical trial are its size and its design. The study design was targeted to the whole spectrum of patients with severe brain injury rather than to those with haematomas and those with diffuse brain injury as distinct groups. The weak findings of adverse effects of hypothermia treatment in patients with diffuse brain injury and the statistically significant finding of improved outcome in patients with evacuated haematomas 
who were treated with hypothermia were each in small numbers of patients. These findings could have been by chance alone.

We see little basis for further testing of early hypothermia induction as a neuroprotectant in diffuse brain injury. However, the role of early hypothermia treatment of patients with evacuated haematomas deserves further testing. In future trials of early hypothermia induction, extending the duration of hypothermia until intracranial pressure levels are low is probably an important strategy to avoid rebound increases in intracranial pressure. Future trials of putative therapies should use different treatment and data analysis strategies for patients with haematomas and diffuse injury.

\section{Acknowledgments}

This study was supported by a grant (5U01 NS043353-06) from the National Institute of Neurological Disorders and Stroke.

\section{References}

1. Sydenham E, Roberts I, Alderson P. Hypothermia for traumatic head injury. Cochrane Database Syst Rev. 2009; 2:CD001048. [PubMed: 19370561]

2. Jiang JY, Yu MK, Zhu C. Effect of long-term mild hypothermia therapy in patients with severe traumatic brain injury: 1-year follow-up review of 87 cases. J Neurosurg. 2000; 93:546-49. [PubMed: 11014530]

3. Jiang JY, Xu W, Li WP, et al. Effect of long-term mild hypothermia or short-term mild hypothermia on outcome of patients with severe traumatic brain injury. J Cereb Blood Flow Metab. 2006; 26:771-76. [PubMed: 16306933]

4. Qiu W, Zhang Y, Sheng H, et al. Effects of therapeutic mild hypothermia on patients with severe traumatic brain injury after craniotomy. J Crit Care. 2007; 22:229-36. [PubMed: 17869973]

5. Yan Y, Tang W, Den Z, et al. Cerebral oxygen metabolism and neuroeletrophysiology in a clinical study of severe brain injury and mild hypothermia. J Clin Neurosci. 2010; 17:196-200. [PubMed: 20036549]

6. Shiozaki T, Nakajima Y, Taneda M, et al. Efficacy of moderate hypothermia in patients with severe head injury and intracranial hypertension refractory to mild hypothermia. J Neurosurg. 2003; 99:4751. [PubMed: 12854743]

7. Clifton GL, Miller ER, Choi SC, et al. Lack of effect of induction of hypothermia after acute brain injury. N Engl J Med. 2001; 344:556-63. [PubMed: 11207351]

8. Marion DW, Penrod E, Kelsey SF, et al. Treatment of traumatic brain injury with moderate hypothermia. N Engl J Med. 1997; 336:540-46. [PubMed: 9023090]

9. Shiozaki T, Hayakata T, Taneda M, et al. A multicenter prospective randomized controlled trial of the efficacy of mild hypothermia for severely head injured patients with low intracranial pressure. $\mathrm{J}$ Neurosurg. 2001; 94:50-54. [PubMed: 11147897]

10. Clifton GL, Miller ER, Choi SC, et al. Hypothermia on admission in patients with severe brain injury. J Neurotrauma. 2002; 19:293-301. [PubMed: 11939497]

11. American Medical Association Committee on Medical Aspects of Automotive Safety. Rating the severity of tissue damage: 1 : the abbreviated scale. JAMA. 1971; 215:277-80. [PubMed: 5107365]

12. Wang HE, Callaway CW, Peitzman AB, Tisherman SA. Admission hypothermia and outcome after major trauma. Crit Care Med. 2005; 33:1296-1301. [PubMed: 15942347]

13. Bratton SL, Chestnut RM, Ghajar J, et al. Guidelines for the management of severe traumatic brain injury: I: hyperventilation. J Neurotrauma. 2007; 24 (suppl 1):S87-90. [PubMed: 17511553]

14. Baker SP, O’Neill B, Haddon W Jr, Long WB. The injury severity score: a method for describing patients with multiple injuries and evaluating emergency care. J Trauma. 1974; 14:187-96.

[PubMed: 4814394] 
15. Myles GL, Perry AG, Malkoff MD, Shatto BJ, Scott-Killmade MC. Quantifying nursing care in barbiturate induced coma with the therapeutic intervention scoring system. J Neurosci Nurs. 1995; 27:35-42. [PubMed: 7769326]

16. Maset AM, Marmarou A, Ward JD, et al. Pressure-volume index in head injury. J Neurosurg. 1987; 67:832-40. [PubMed: 3681422]

17. Hutchison JS, Ward RE, Lacroix J, et al. Hypothermia Pediatric Head Injury Trial Investigators and the Canadian Critical Care Trials Group. Hypothermia therapy after traumatic brain injury in children. N Engl J Med. 2008; 358:2447-56. [PubMed: 18525042]

18. Valadka AB, Gopinath SP, Contant CF, Uzura M, Robertson CS. Relationship of brain tissue PO2 to outcome after severe head injury. Crit Care Med. 1998; 26:1482-84. [PubMed: 9751580]

19. Miller JD, Becker DP, Ward JD, Sullivan HG, Adams WE, Rosner MJ. Significance of intracranial hypertension in severe head injury. J Neurosurg. 1977; 47:503-16. [PubMed: 903804]

20. Kurodo Y, Bullock R. Local cerebral blood flow mapping before and after removal of acute subdural hematoma in the rat. Neurosurgery. 1992; 30:687-91. [PubMed: 1316565]

21. Janata A, Holzer M. Hypothermia after cardiac arrest. Prog Cardiovasc Dis. 2009; 52:168-79. [PubMed: 19732608]

22. Burger R, Bendszus M, Vince GH, Solymosi L, Roosen K. Neurophysiological monitoring, magnetic resonance imaging, and histological assays confirm the beneficial effects of moderate hypothermia after epidural focal mass lesion development in rodents. Neurosurgery. 2004; 54:701-12. [PubMed: 15028147]

23. Farkas O, Povlishock JT. Cellular and subcellular change evoked by diffuse traumatic brain injury: a complex web of change extending far beyond focal damage. Prog Brain Res. 2007; 161:43-59. [PubMed: 17618969] 


\section{Panel: Research in context}

\section{Systematic review}

We reviewed manuscripts that were referenced in a recent Cochrane review of hypothermia for treatment of severe brain injury, ${ }^{1}$ which included 23 clinical trials involving 1614 patients. Eight of these trials were done in adults, applied systemic rather than head cooling, were written in English, were not specified as phase 2 trials or pilot trials, were not small trials (included 17-28 patients), did not have biochemical alterations as the primary outcome, and reported neurological outcomes. Five of the eight clinical trials that met these criteria extended hypothermia for 3-5 days or until intracranial hypertension resolved. ${ }^{2-6}$ Four of these five clinical trials reported improved outcome, ${ }^{2-5}$ accompanied in three ${ }^{2-4}$ by significantly decreased intracranial pressure with hypothermia treatment. Three of eight trials induced hypothermia within $10 \mathrm{~h}$ of injury for 1-2 days and rewarmed regardless of raised intracranial pressure. ${ }^{7-9}$ None of these trials reported a treatment effect of hypothermia.

\section{Interpretation}

Clinical trials of reasonable quality support the use of hypothermia for an extended period of time for control of intracranial pressure in patients with severe brain injury. There is no evidence that early induction of hypothermia as a neuroprotectant for 1-2 days improves outcome. When taken in the context of other clinical trials, the results of this study provide little justification for further studies of early hypothermia as a neuroprotectant in patients with diffuse brain injury. However, the finding of an improved outcome in patients with evacuated haematomas with very early hypothermia induction deserves further study. 


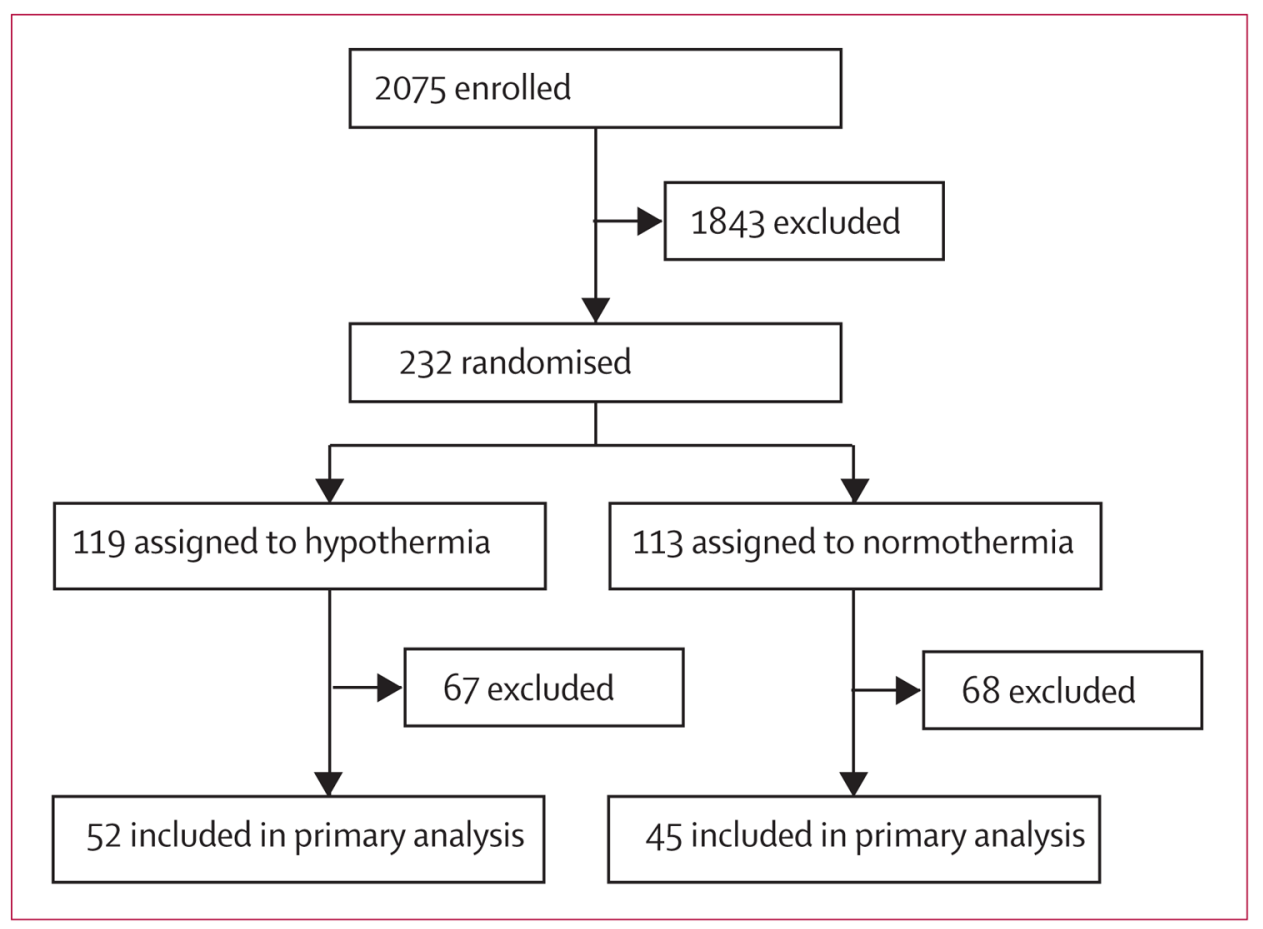

Figure 1.

Trial profile 


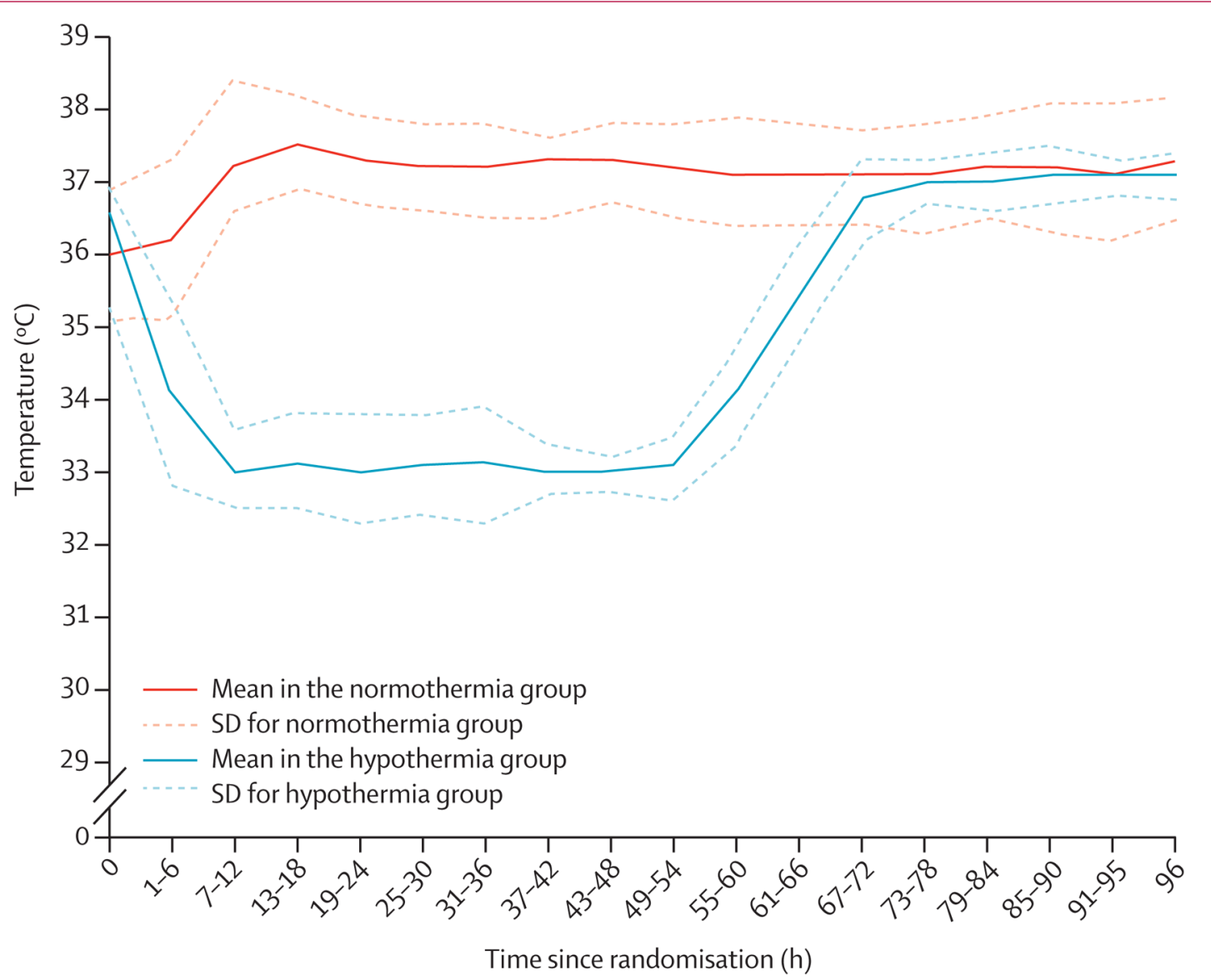

Number at risk

$\begin{array}{lllllllllllllllllll}\text { Hypothermia } & 52 & 52 & 52 & 52 & 51 & 51 & 49 & 48 & 49 & 48 & 47 & 47 & 45 & 46 & 45 & 45 & 45 & 43\end{array}$ $\begin{array}{lllllllllllllllllll}\text { Normothermia } & 45 & 45 & 45 & 44 & 44 & 45 & 44 & 44 & 44 & 44 & 44 & 42 & 42 & 42 & 41 & 40 & 37 & 35\end{array}$

Figure 2. Temperature analysis during the first $96 \mathrm{~h}$ after randomisation The number of patients decreased over time as patients died or were discharged from intensive care. 
Table 1

Demographics and baseline characteristics

\begin{tabular}{|c|c|c|}
\hline & Hypothermia $(n=52)$ & Normothermia $(n=45)$ \\
\hline Age (years) & $26(9)$ & $31(11)$ \\
\hline GCS score 5-8 & $33(63 \%)$ & $22(49 \%)$ \\
\hline GCS score 3-4 & $19(37 \%)$ & $23(51 \%)$ \\
\hline Non-reactive pupils ${ }^{*}$ & $6(12 \%)$ & $5(11 \%)$ \\
\hline Surgical lesion removed in first $24 \mathrm{~h}$ after injury & $15(29 \%)$ & $15(33 \%)$ \\
\hline Prehospital hypotension ${ }^{\dagger}$ & $7(15 \%)$ & $7(16 \%)$ \\
\hline Prehospital hypoxia $f$ & $11(23 \%)$ & $4(9 \%)$ \\
\hline Injury severity score & $30(6)$ & $30(9)$ \\
\hline Abbreviated injury severity score for head & $4 \cdot 56(0 \cdot 61)$ & $4.47(0.63)$ \\
\hline Positive blood alcohol $\xi$ & $17(59 \%)$ & $17(59 \%)$ \\
\hline First temperature $\left({ }^{\circ} \mathrm{C}\right) \mathrm{g} /$ & $36 \cdot 1(0 \cdot 8)$ & $36 \cdot 0(0 \cdot 9)$ \\
\hline
\end{tabular}

Data are mean $(\mathrm{SD})$ or number $(\%)$. GCS=Glasgow coma scale.

* Data missing for three patients in the hypothermia group and one in the normothermia group.

Data missing for four patients in the hypothermia group and two in the normothermia group.

${ }^{*}$ Data missing for four patients in the hypothermia group and two in the normothermia group.

$\mathcal{\xi}$ Data missing for 23 patients in the hypothermia group and 16 in the normothermia group.

Il Data missing for one patient in the normothermia group 
Table 3

Factors associated with medical treatment after hospitalisation

\begin{tabular}{|c|c|c|c|}
\hline & Hypothermia (n=52) & Normothermia $(n=45)$ & p value \\
\hline Received morphine & $49(94 \%)$ & $39(87 \%)$ & $0 \cdot 30$ \\
\hline Morphine dose $(\mathrm{mg} / \mathrm{h})^{*}$ & $6 \cdot 5(2 \cdot 4)$ & $6 \cdot 6(3 \cdot 0)$ & $0 \cdot 88$ \\
\hline Received any paralytic & $51(98 \%)$ & $37(82 \%)$ & $0 \cdot 01$ \\
\hline Received pancuronium bromide & $39(75 \%)$ & $26(58 \%)$ & $0 \cdot 07$ \\
\hline Pancuronium bromide dose $(\mathrm{mg} / \mathrm{h})$ & $4.9(2.7)$ & $6 \cdot 8(9 \cdot 0)$ & $0 \cdot 30$ \\
\hline Received mannitol & $39(75 \%)$ & $27(60 \%)$ & $0 \cdot 11$ \\
\hline Mannitol dose (g/day) ${ }^{*}$ & $59(3)$ & $51(60)$ & $0 \cdot 27$ \\
\hline Received phenytoin & $47(90 \%)$ & $42(93 \%)$ & $0 \cdot 72$ \\
\hline Phenytoin dose (mg/day) ${ }^{*}$ & $224(251)$ & $283(311)$ & $0 \cdot 33$ \\
\hline Administered vasopressors $*$ & $42(81 \%)$ & $32(71 \%)$ & $0 \cdot 26$ \\
\hline Daily therapeutic intervention score in ICU ${ }^{*}$ & $46(8)$ & $40(7)$ & 0.0006 \\
\hline Daily therapeutic intervention score after ICU discharge & $17(18)$ & $15(15)$ & $0 \cdot 46$ \\
\hline Daily therapeutic intensity level $* \dagger$ & $5 \cdot 4(2 \cdot 3)$ & $4 \cdot 0(2 \cdot 0)$ & 0.002 \\
\hline Fluids administered $(\mathrm{mL})$ & $2375(1240)$ & $1159(999)$ & $<0.0001$ \\
\hline Cumulative fluid balance $(\mathrm{mL})^{*}$ & $5285(4285)$ & $3099(4080)$ & $0 \cdot 01$ \\
\hline Negative fluid balance ${ }^{*}$ & $4(8 \%)$ & $9(20 \%)$ & 0.08 \\
\hline Caloric intake on day 5 (kcal) & $761(732)$ & $704(800)$ & $0 \cdot 71$ \\
\hline
\end{tabular}

Data are mean $(\mathrm{SD})$ or number $(\%)$. ICU=intensive care unit.

* For the first $96 \mathrm{~h}$ after injury.

${ }^{\dagger}$ Calculated for treatments administered to each patient in each 24-h period and mean values over 4 days were taken for each patient. 
Table 4

Laboratory findings

\begin{tabular}{|c|c|c|c|}
\hline & Hypothermia $(n=52)$ & Normothermia $(n=45)$ & p value \\
\hline Serum sodium $(\mathrm{mmol} / \mathrm{L})$ & $143(5)$ & $143(6)$ & $0 \cdot 80$ \\
\hline Serum potassium $(\mathrm{mmol} / \mathrm{L})$ & $3 \cdot 6(0 \cdot 3)$ & $3 \cdot 8(0 \cdot 2)$ & 0.0005 \\
\hline Blood urea nitrogen $(\mathrm{mmol} / \mathrm{L})$ & $4 \cdot 3(2 \cdot 9)$ & $3.9(1 \cdot 4)$ & $0 \cdot 28$ \\
\hline Serum creatinine $(\mu \mathrm{mol} / \mathrm{L})$ & $74(36)$ & $72(18)$ & $0 \cdot 66$ \\
\hline Prothrombin time $(\mathrm{s}){ }^{*}$ & $15(2)$ & $14(3)$ & $0 \cdot 73$ \\
\hline Partial thromboplastin time $(\mathrm{s})^{\dagger}$ & $34(7)$ & $31(5)$ & $0 \cdot 004$ \\
\hline Haemoglobin $(\mathrm{g} / \mathrm{L})$ & $110(10)$ & $110(10)$ & $0 \cdot 28$ \\
\hline Platelet count (cells per $\mu \mathrm{L})^{* t}$ & $189(77)$ & $209(74)$ & $0 \cdot 2$ \\
\hline Serum magnesium $(\mathrm{mmol} / \mathrm{L})^{\xi}$ & $0 \cdot 8(0 \cdot 1)$ & $0 \cdot 8(0 \cdot 1)$ & $0 \cdot 46$ \\
\hline Partial pressure of brain oxygen $<6 \mathrm{~mm} \mathrm{Hg}$ & $19(39 \%)$ & $18(45 \%)$ & $0 \cdot 55$ \\
\hline Partial pressure of arterial carbon dioxide $(\mathrm{mm} \mathrm{Hg}) \mathscr{q}$ & $36(4)$ & $37(3)$ & $0 \cdot 75$ \\
\hline Partial pressure of arterial carbon dioxide $<30 \mathrm{~mm} \mathrm{Hg}$ & $43(83 \%)$ & $28(62 \%)$ & $0 \cdot 02$ \\
\hline Serum glucose $(\mathrm{mmol} / \mathrm{L})$ & $7 \cdot 6(2 \cdot 0)$ & $7 \cdot 1(0 \cdot 8)$ & $0 \cdot 12$ \\
\hline
\end{tabular}

Data are mean (SD) or number (\%). Mean values were calculated for each patient who had a value recorded from the time of admission until discharge from the intensive care unit. Mean values are not corrected for the duration of intensive care stay or the number of values collected for each patient.

Data missing for 11 patients in the hypothermia group and 12 in the normothermia group.

${ }^{t}$ Data missing for two patients in the hypothermia group.

${ }^{*}$ Data missing for two patients in the hypothermia group.

$\xi_{\text {Data missing for one patient in the hypothermia group. }}$

I/ Not corrected for temperature (alpha stat). 


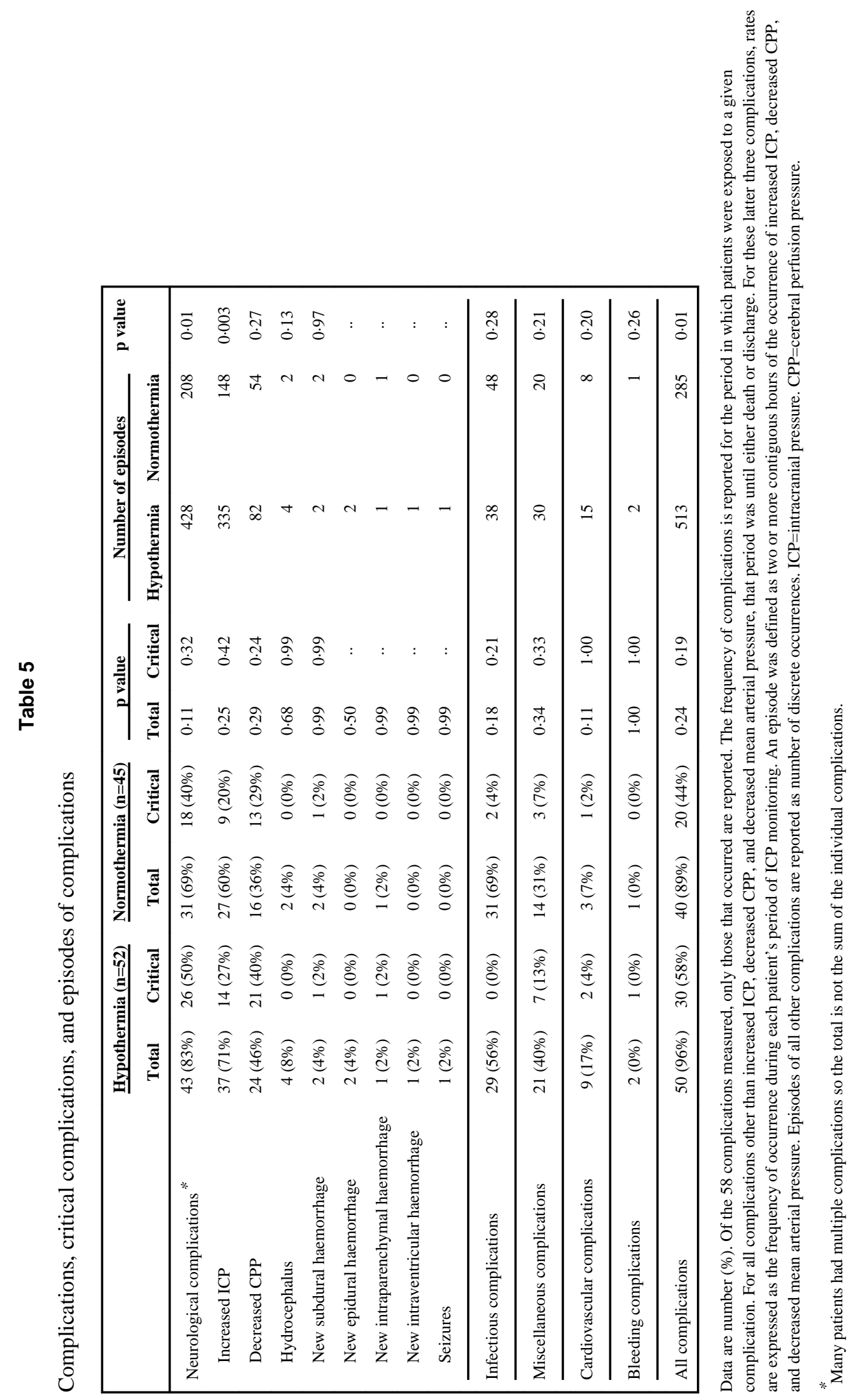

